\title{
Initial Study on Implementation of the Low-Frequency Wave Markers for the Purpose of Diagnostic Tests' Performance and Neurofeedback Therapy
}

\author{
Magda Zolubak ${ }^{1,4}$, Mariusz Pelc ${ }^{1,2}$, Stepan Ozana ${ }^{3}$, \\ Krystyna Macek-Kamińska ${ }^{1}$, Barbara Grochowicz ${ }^{1}$, \\ Aleksandra Kawala-Sterniuk ${ }^{1}$
}

${ }^{1}$ Faculty of Electrical Engineering, Automatic Control and Informatics, Opole University of Technology, Poland

2 Department of Computing and Information Systems, University of Greenwich, United Kingdom

3 Department of Cybernetics and Biomedical Engineering, VSB Technical University Ostrava, Czech Republic

${ }^{4}$ Faculty of Mathematics, Physics and Computer Science, University of Opole, Poland

\begin{abstract}
The paper focuses on automation of signal processing, which also considers analysis of biomedical data, such as EEG. The results of the study prove that this enables a better understanding of signal changes and makes it possible to address some specific disturbances. It also makes it possible to describe the relevant changes in signals mathematically and helps to create markers of various brain disorders. This paper presents the study at the initial stage and focuses on the mathematical markers of concentration disorders associated with Theta waves. The presented markers presented are based on Welch's periodograms. The obtained results are very promising and further studies aimed at refining them are currently being carried out.
\end{abstract}

\section{Introduction}

This paper presents the initial stage of a study focused on potential electroencephalography signal analysis, automation of various processes of signal analysis, and processing of biomedical data such as EEG. It enables a better understanding of signal changes and allows to name some specific disturbances. It also makes it possible to describe the relevant changes in signals mathematically and create markers of various brain disorders. The presented study is still at the initial stage and focuses on mathematical markers of concentration disorders associated with Theta waves. The markers pre- 
sented here are based on Welch's periodograms (Sierra-Alonso et al., 2019; Welch, 1967).

Classification of biomedical data is a problem due to the nature of such signals, therefore this task is very challenging; however, classification of spectral power features of EEG data is currently quite well documented. Proper data-classification plays a crucial role in diagnostics and later in choosing the appropriate therapy (Kawala-Janik et al., 2018; Lazar et al., 2019; Martinez-Murcia et al., 2019).

The method described in this paper is based on periodograms, which are one of the Power Spectral Density (PSD) estimators, first applied by Arthur Schuster as early as in 1898. PSD can be defined as the magnitude squared of the Fourier transform of the measured signal's autocorrelation function. Both the mean and variance enable to characterize the PSD estimator (Bartlett, 1950; Rooney \& Buck, 2019; Sierra-Alonso et al., 2019; Welch, 1967).

Various brain disorders such as Attention Deficit Disorder (ADD) or Attention Deficit Hyperactivity Disorder (ADHD) are becoming more common nowadays, affecting about $5 \%$ of children and adolescents and about 2.5\% of adults (Kostick, 2017; World Health Organization, 2015; Asherson et al. 2018). Due to the character of these disorders it is important to adapt the potential therapy individually to the patient's needs. For this reason, one of the increasingly popular therapy methods is Neurofeedbackbased therapy (Budzynski et al., 2009).

\section{Research Methodology}

The data was recorded at a frequency range of $1-70 \mathrm{~Hz}$. The sampling frequency was $250 \mathrm{~Hz}$. The amplifier used for the study was a DigiTrack BF type digital electroencephalograph. The device registers EEG signal in the time domain. In addition, a program dedicated to this device provides QEEG (Quantitative Electroencephalography) analysis. The measuring equipment consisted of a reference electrode, a cup-shaped electrode, measurement interfaces, and a transducer used for analyses/signal pre-processing. Figure 1 shows the measuring equipment.

The Neurofeedback-based therapy in ADHD treatment is still a controversial method compared to pharmacological or behavioral ones; however, it is becoming more and more popular due to the increasing number of publications describing its positive results (Bluschke et al., 2016; MorenoGarcia et al., 2015; Moreno-Garcia et al., 2019). 


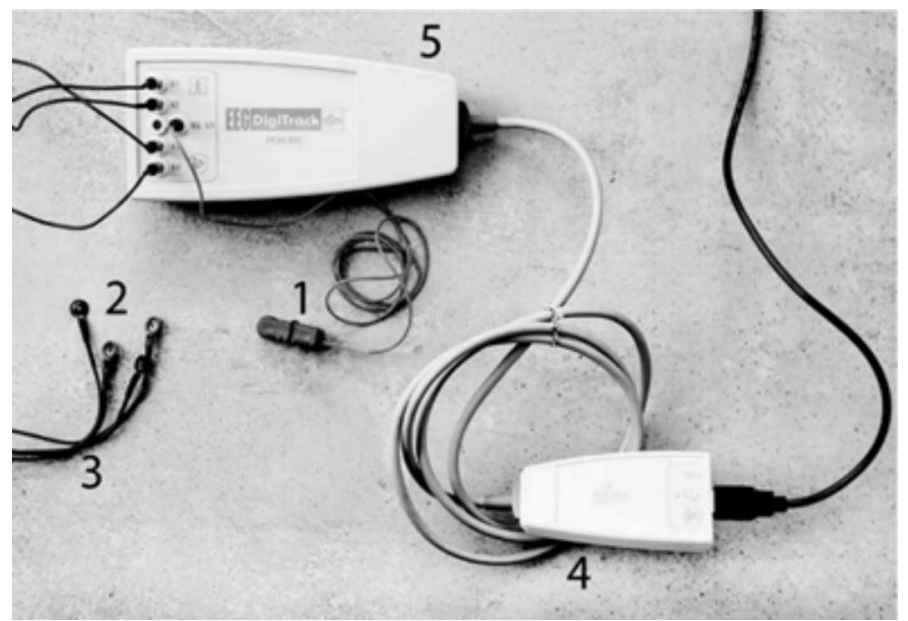

Figure 1. The measuring equipment: 1 - reference electrode, 2 - cup-shaped electrode, 3 - wiring, 4 -measurement interfaces, 5 -transducer for analyses/signal pre-processing

The vast body of literature in the Neurofeedback field suggests that specific bandwidths are associated with particular cognitive functions. Deficits or excesses in these frequencies constitute the basis for the diagnosis of certain disorders. For example, it is possible to notice the occurrence of reduced sensorimotor rhythm in the central strip and excess slow wave activity at the front of the head in children with ADHD. Furthermore, in children with ADD, apart from reduced sensorimotor rhythm, distortions in Beta frequency and adjacent excess Alpha activity was observed in quantitative analyses (Hartmut et al., 2016; Schmid et al., 2018; Schonenberg et al., 2017).

In neuropsychology and neurobiology, sensorimotor rhythm is usually associated with external attention and relaxation. In the case of reduced sensorimotor rhythm, symptoms such as tensions in attention, concentration difficulties, or even low mood may occur (Hartmut et al., 2016; Skirrow et al., 2015; Wilhelm et al., 2014).

Based on the authors' professional experience and in-depth literature study, it can be stated that Theta- and Beta-based protocols are usually used in the front of the head or the central line, depending on the patient's needs (Bluschke et al., 2016; Mehran et al., 2015; Schlerf et al., 2015; Schmid et al., 2018; Schonenberg et al., 2017). As the Theta frequency (4$8 \mathrm{~Hz}$ ) can be recorded mainly in the front of the head, analysis of its values obtained from the ' $\mathrm{Fz}$ ', 'C3', and ' $\mathrm{C} 4$ ' channels seems to be the most useful in the diagnosis of ADD and ADHD. Diagnostics based on EEG signal 
analysis can be performed as a signal analysis in the time domain or after transfer of the recording to the frequency domain (Clarke et al., 2019; Saad et al., 2018; Schonenberg et al., 2017).

For digital analysis, the EEG signal is usually transferred from the time domain to the frequency domain, as mentioned above. These can be various transformations, including the Fast Fourier Transform (FFT) or periodograms. This allows to extract more information from the signal. The application of the power density spectrum (PSD) may give different results depending on the applied method. Among the various methods popular in the PSD analysis is the Least-Squares Spectral Analysis and the simplified Welch's periodogram. Both methods are similar to the Fourier Transform (FT) and the Fast Fourier Transform (FFT). When analyzing the signal in the low frequency range, it is important to properly select the appropriate filtering method before performing the PSD analysis (Bakhshi et al., 2013; Kubacki et al., 2016).

The authors decided to implement the Welch's periodogram method, which is an improved version of the typical (standard) periodogram (Martinez-Murcia et al., 2019; Mostile et al., 2019; Mulkey et al., 2019). The chosen method is the modified periodogram, which is an improved estimator of the Power Spectral Density (PSD) consisting of the time-series divided into segments. The PSD estimates are then averaged and the result is Welch's PSD estimate (Welch, 1967).

The described method relies on the division of length $L$ into segments $K$, which overlap in points $D$, where parameter $S$ is the number of new points in each segment. The number of points common to the two neighboring segments is $M$-S. If $M=S$, then the segment overlap is $0 \%$; however, if $S=0.5 M$, then the segment overlap is $50 \%$.

As the Welch's method can cause losses at the signal limits, to compensate them, the segments are often overlapped in the time domain. Therefore, windowing the signal makes this method a modification of the classic periodogram (Welch, 1967; Zhou et al., 2016).

\section{The Conducted Experiments}

The authors decided to use Quantitative EEG (QEEG) (Budzynski et al., 2009) in the study. The electrodes were placed above the motor cortex area in the following locations: 'Fz', 'C3', 'C4', and ' $\mathrm{Cz}$ ', in accordance with the '10-20' system (Homan et al., 1987). In Figure 2 shows the '10-20' system with marked measurement points (Kawala-Janik et al., 2018). 


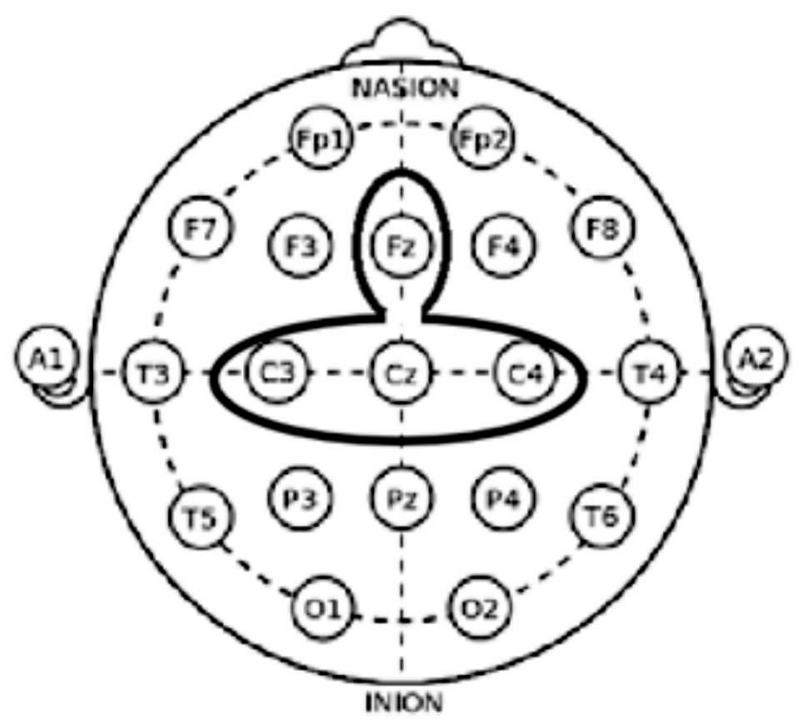

Figure 2. Measurement points (motor cortex area) (Kawala-Janik et al., 2018)

In order to determine the markers for attention disorders associated with the low frequency waves, five boys aged under $14(N=5)$ were tested. All the subjects were diagnosed by a neurologist who detected problems with concentration due to ADD-like disorders. Repetitive changes were observed in recordings obtained from the participants. Based on the changes visible in the QEEG analysis, markers of disorders were determined from Welch's periodograms. In the EEG signal, the Theta frequency is seen as equal amplitude.

\section{Results and Discussion}

In the study, 14 subjects were tested together with a small healthy control group (one healthy participant) in order to compare the obtained results and to confirm the data regarding markers. Based on the results, two types of disorders were identified, named I and II.

Figure 3 shows an example of excess Theta waves. The rectangle shows excess low waves (Theta), visible as 5 amplitudes recorded within one second. Figure 4 presents a recording of the signal from channels ' $\mathrm{Cz}$ ' and 'C3' shown in Figure 3 as a spectrogram. The recording was obtained from a patient with ADD. 
Magda Zolubak et al.

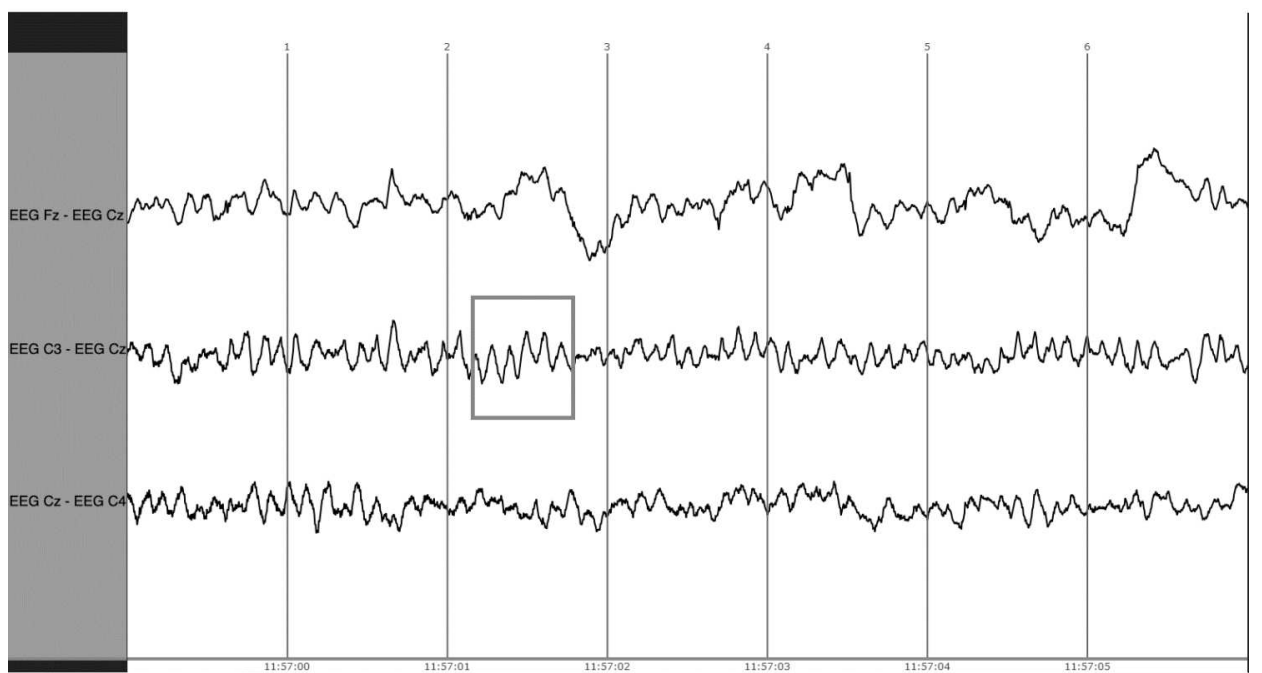

Figure 3. Excess Theta waves - sample 1
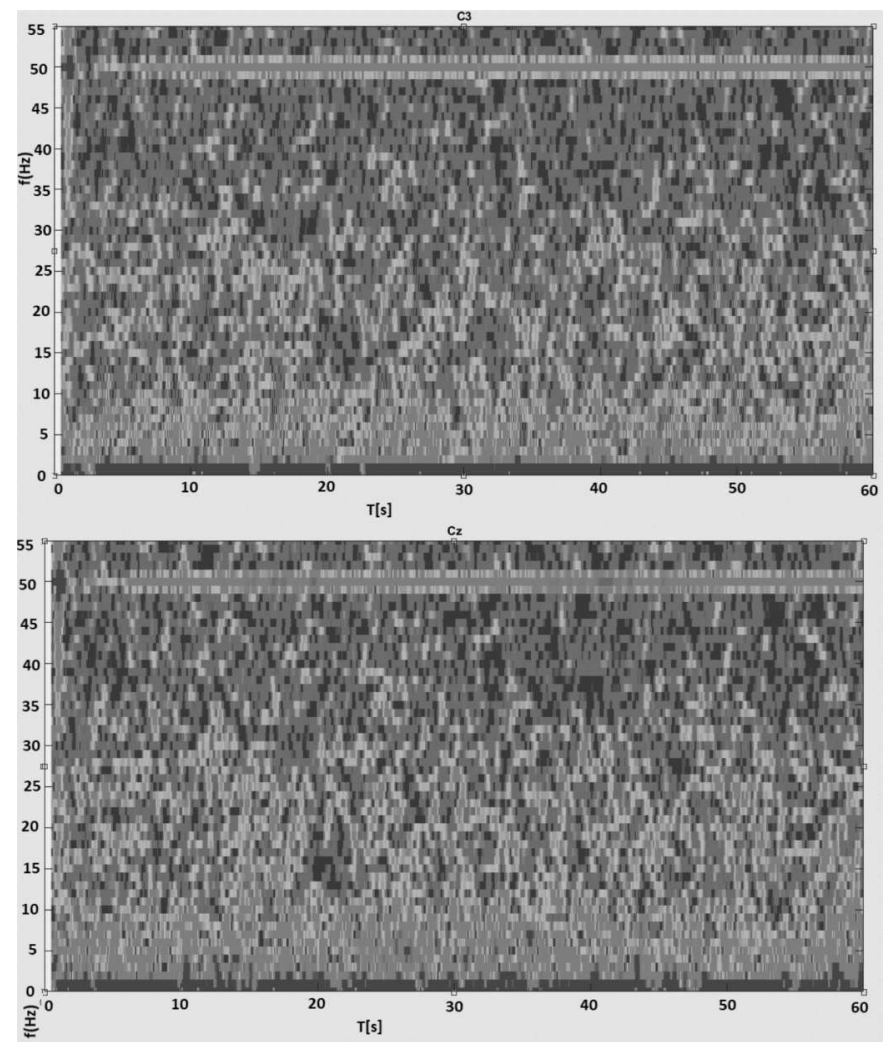

Figure 4. Example of excess Theta waves - spectrogram, sample 1 
The results can be presented in a legible form as a matrix with all the important values. In this case, the output is a matrix consisting of 4 lines and 7 columns. The lines are corresponding to the electrodes used for the measurements: 'Fz', 'Cz', 'C3', 'C4', where the columns are associated with brainwave frequencies: 'Delta', 'Theta', 'Alpha', 'SMR', 'Beta1', 'Beta2', and 'Gamma'. ' $\mathrm{X}$ ' and ' $\mathrm{Y}$ ' describe the coordinates of the periodogram. Figure 5 presents a sample matrix obtained from the four ('Fz', 'Cz', 'C3', 'C4') measurement points after the Welch's PSD analysis was applied. The matrix contains the maximum coordinates.

\begin{tabular}{|c|c|c|c|c|c|c|c|}
\hline $\mathbf{F z}$ & $\begin{array}{c}\text { Delta } \\
0.1221\end{array}$ & $\begin{array}{l}\text { Theta } \\
4.2725\end{array}$ & $\begin{array}{l}\text { Alpha } \\
10.2539\end{array}$ & $\begin{array}{l}\text { SMR } \\
14.4043\end{array}$ & $\begin{array}{c}\text { Beta1 } \\
17.9443\end{array}$ & $\begin{array}{l}\text { Beta2 } \\
27.8320\end{array}$ & $\begin{array}{l}\text { Gamma } \\
34.1797\end{array}$ \\
\hline$c z$ & 0.1221 & 4.3945 & 10.2539 & 14.0381 & 17.9443 & 26.9775 & 34.1797 \\
\hline C3 & 0.1221 & 4.3945 & 10.2539 & 13.6719 & 18.3105 & 26.7334 & 36.2549 \\
\hline C4 & 2.3193 & 4.2725 & 10.2539 & 14.1602 & 17.9443 & 26.9775 & 34.5459 \\
\hline \multicolumn{8}{|l|}{$\mathrm{Y}=$} \\
\hline$F_{z}$ & $\begin{array}{r}\text { Delta } \\
3.7147\end{array}$ & $\begin{array}{l}\text { Theta } \\
3.3540\end{array}$ & $\begin{array}{l}\text { Alpha } \\
2.4438\end{array}$ & $\begin{array}{l}\text { SMR } \\
-0.0284\end{array}$ & $\begin{array}{l}\text { Beta1 } \\
3.1189\end{array}$ & $\begin{array}{l}\text { Beta2 } \\
2.5477\end{array}$ & $\begin{array}{l}\text { Gamma } \\
1.2064\end{array}$ \\
\hline$C_{2}$ & 2.0254 & 2.8370 & 3.8038 & -0.7568 & 3.2497 & 3.0025 & 0.7625 \\
\hline C3 & 2.7592 & 3.5266 & 4.2134 & 0.1747 & 1.5153 & 2.5887 & 2.1758 \\
\hline$C 4$ & 2.7776 & 2.9616 & 3.9213 & -0.4163 & 3.2182 & 2.6258 & 0.9812 \\
\hline
\end{tabular}

Figure 5. Sample matrix

Figures 6-9 illustrate further sample matrices with the obtained values and PSDs for subjects with attention disorders. It can be noticed that they are strongly connected with the low frequency present in signals.

$\mathrm{x}=$

\begin{tabular}{|c|c|c|c|c|c|c|}
\hline 0.2441 & 6.9580 & 9.1553 & 13.5498 & 16.8457 & 24.1699 & 35.8887 \\
\hline 3.9063 & 7.3242 & 8.6670 & 13.5498 & 16.8457 & 20.8740 & 39.5508 \\
\hline 0.1221 & 6.8359 & 9.1553 & 12.0850 & 17.3340 & 22.2168 & 39.3066 \\
\hline 3.9063 & 7.8125 & 8.6670 & 12.2070 & 16.3574 & 20.3857 & 39.5508 \\
\hline
\end{tabular}

$\mathrm{Y}=$

$\begin{array}{rrrrrrr}3.6884 & 3.9243 & 3.0032 & 1.5528 & -0.6765 & -1.2156 & -1.0840 \\ 3.0615 & 4.5907 & 3.9489 & 1.2512 & -0.7270 & -2.9122 & -0.8763 \\ 3.8105 & 2.1843 & 5.0554 & 2.3835 & -0.0468 & -2.4474 & -0.1952 \\ 4.0862 & 5.3880 & 6.1623 & 3.1570 & 0.6886 & -2.0506 & -0.3645\end{array}$

Figure 6. Matrix - low frequencies, attention disorders, sample 1 


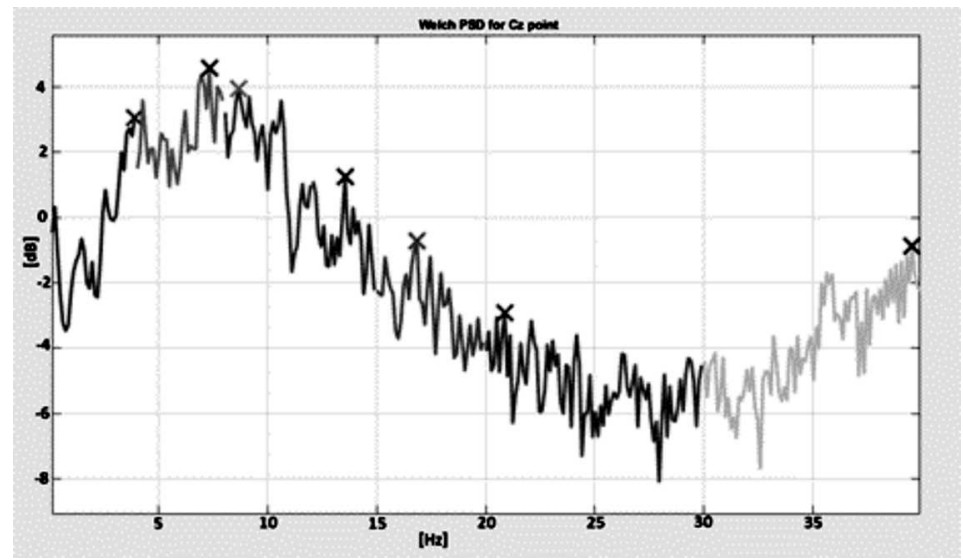

Figure 7. PSD - low frequencies, attention disorders, sample 1

$x=$

3.6621

0.2441

1.0986

0.2441

5.6152
5.7373
5.6152
6.2256

9.2773

12.4512

18.5547

29.4189

32.3486

8.9111

13.1836

18.4326

29.4189

37.4756

9.3994

13.1836

19.5313

28.1982

38.3301

9.2773

12.4512

17.9443

29.4189

38.3301

$\mathrm{Y}=$

\begin{tabular}{rrr|r|rrr}
-0.0413 & 3.3910 & 6.5951 & 0.4751 & 1.3278 & -0.1790 & -0.3426 \\
5.5218 & 3.2176 & 8.0526 & -0.3771 & 0.7917 & -1.0913 & -0.8706 \\
7.1662 & 3.7992 & 10.3846 & -1.7548 & 0.1515 & -0.3870 & -0.9713 \\
4.5198 & 3.1308 & 8.8017 & 0.6637 & 0.6436 & -0.8662 & -0.2479
\end{tabular}

Figure 8. Matrix - low frequencies, attention disorders, sample 2

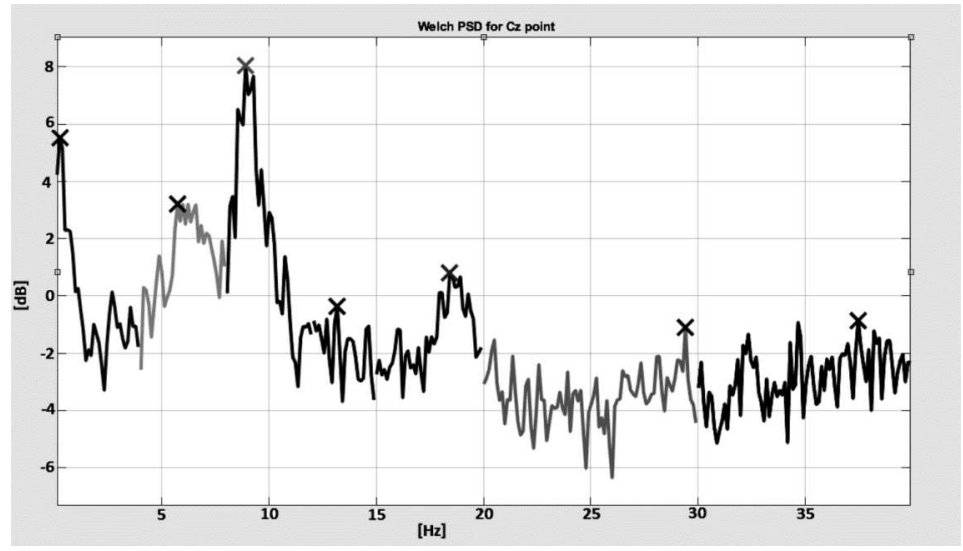

Figure 9. PSD - low frequencies, attention disorders, sample 2 
Initial Study on Implementation of the Low-Frequency Wave Markers...

As mentioned above, it is possible to classify disorders into two types, i.e. type I and type II. Type I is excess Theta frequency; in all the cases the maximum is above $5.5 \mathrm{~Hz}$. Type II is also excess Theta frequency; in all cases the maximum is above $5.5 \mathrm{~Hz}$ and the SMR value is below $1 \mathrm{~dB}$.

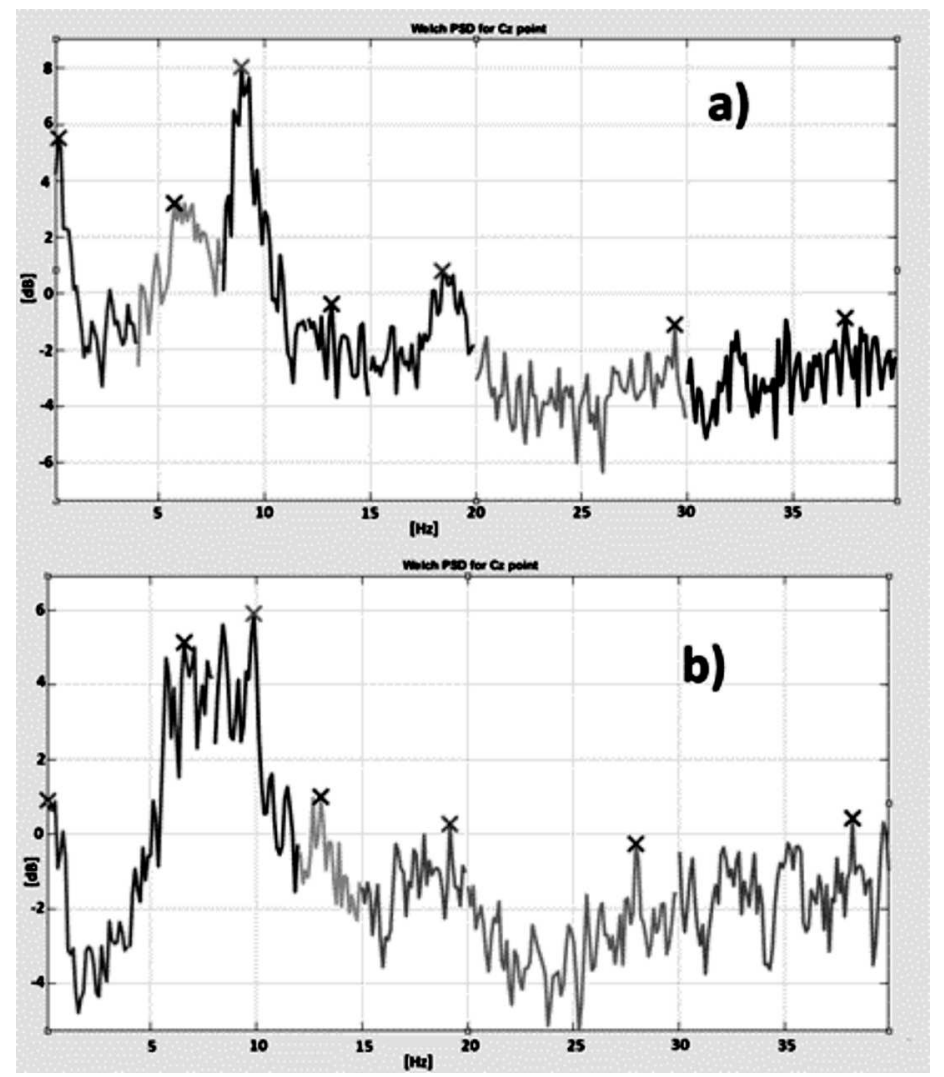

Figure 10. Spectrograms before (a) and after the Neurofeedback therapy (b) for type II

Figure 10 shows the results before (a) and after therapy (b). An improvement in the overall functioning of the subjects and a reduction of the level of stress can be observed. Moreover, the SMR equaled the normal values. Unfortunately, the Theta values were still too high. Such results suggest a continuation of the training provided for type II disorder.

When analyzing the data for one of the boys, a low SMR, below $1.5 \mathrm{~dB}$, was observed in 3 points. What is more, a higher level of stress could easily be observed. Such results may suggest the possibility of distinguishing a third type of attention disorders. Figure 11 shows a matrix with the recorded results. 
Magda Zolubak et al.

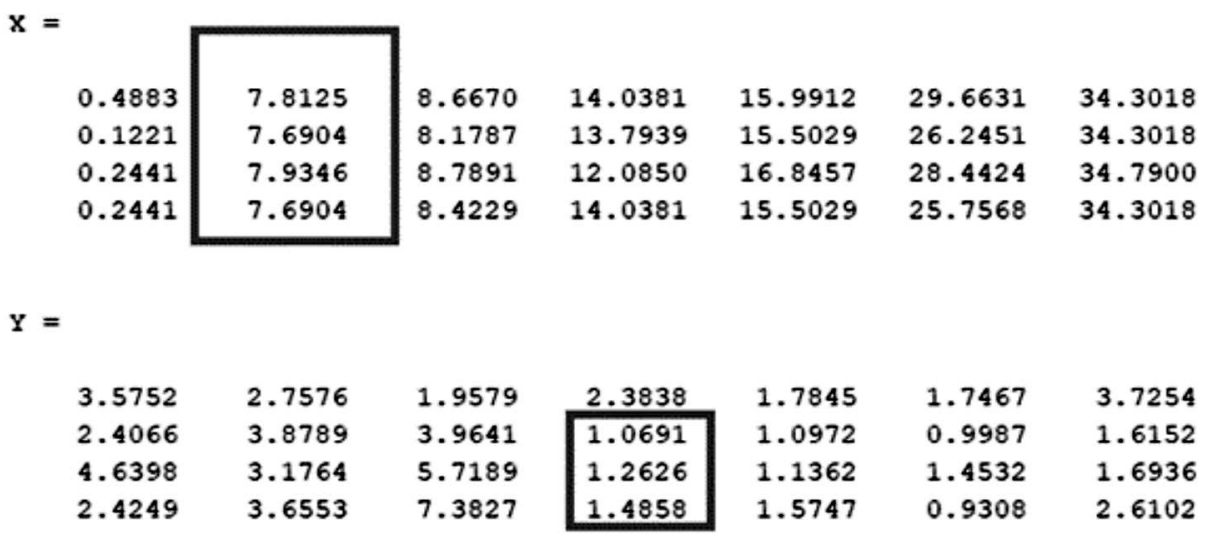

Figure 11. Sample matrix suggesting type III disorder

Figure 12 shows the PSD for the intermediate type between the first and the second type. Finally, this type was assigned to type I, with SMR above $1 \mathrm{~dB}$. In the authors' opinion, the use of diagnostic markers based on Welch's analysis and co-operative maximum values enables as good diagnostics as the currently used methods. These are, however, a much faster diagnostic method which also allows to recognize the cause of the problem.

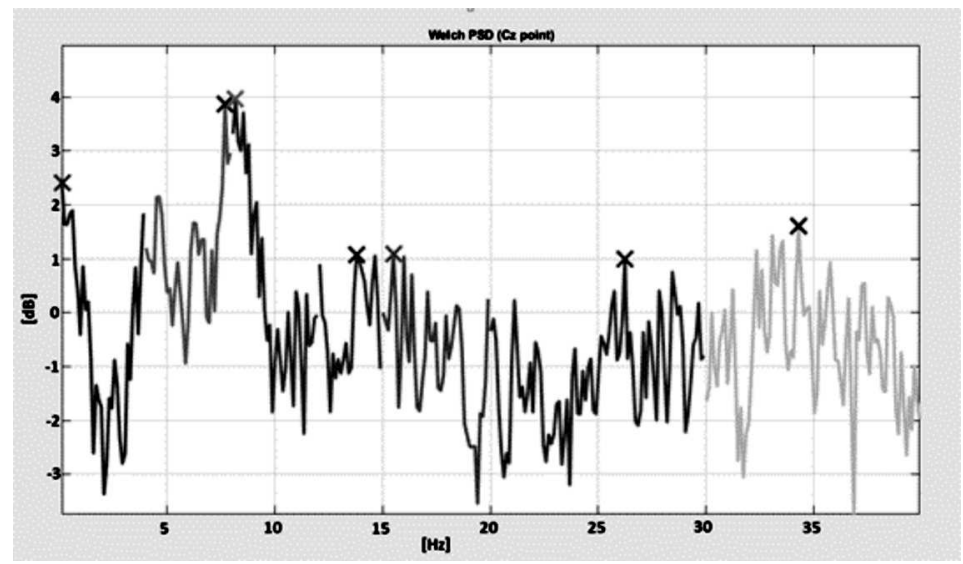

Figure 12. Welch's PSD

Figure 13 shows two types of ADD/ADHD, providing information obtained by analyzing the data using the marker method described above. The two types of markers enable to present the information in question, i.e. Type I with excess Theta only and Type II with low SMR and excess Theta. 
Initial Study on Implementation of the Low-Frequency Wave Markers...
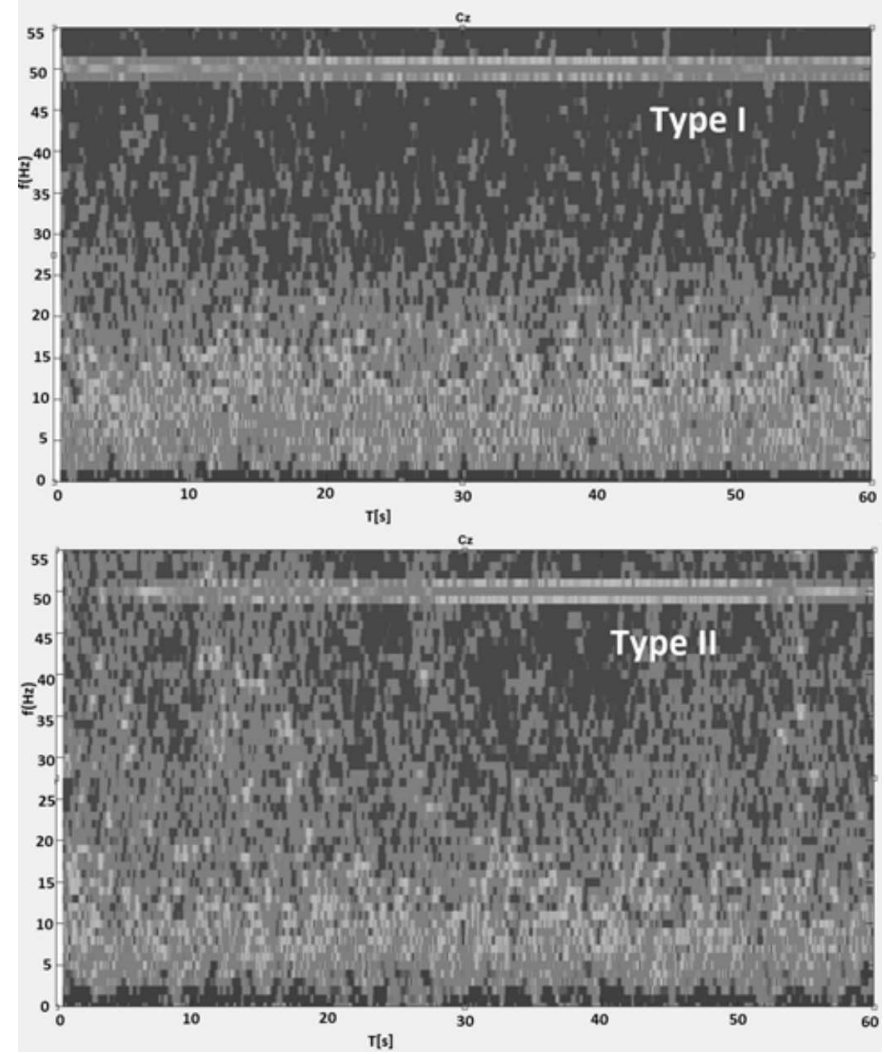

Figure 13. Two types of disorders (type I and II) - markers

All the participants who took part in the study were treated with the Neurofeedback therapy. After a series of 10 trainings, they were subjected to follow-up examinations in order to check the improvement. Next, the training protocols used were compared to the obtained results for the markers method. The results showed a relationship between the training protocols $\mathrm{SMR} / \mathrm{Theta} /$ Beta in the central line and type II disorders. In the case of type I disorder - the training protocols, SMR/Theta in the non-dominant hemisphere, affected the results for the right and the left hemisphere. In order to confirm the obtained results, the signals were additionally analyzed using spectrograms.

Since the markers are to target a specific type of disorder and to facilitate diagnosis and therapy, the modified periodogram method is sufficient. The conducted research proved a dependence between the type of Neurofeedback therapy and type I and II disorders, which suggests the need for standardization of changes in the applied Neurofeedback training protocols. 


\section{Final Remarks}

In further studies, it is necessary to examine a greater number of children with various concentration disorders. This will help to develop more algorithms that may classify changes present in signals. It would be useful to analyze the training protocols used in children with disorders associated with low frequency waves. The authors are planning to develop algorithms for the therapy of ADD and ADHD disorders.

The second low frequency, i.e. Delta wave, is usually the largest to observe in locations ' $\mathrm{Fz}$ ' and ' $\mathrm{C} 3$ '. Based on literature study, data from those locations is mostly analyzed with regards to testing children with ADD and ADHD. Unfortunately, the Delta wave also increases during movement during the test, so in further analyzes it will be important to choose , e.g., a smoothing filter that will allow to create another marker, one that may be based on slow waves such as Delta and Theta, instead of on Theta or Theta/SMR only (Saad et al., 2018).

Creating new markers based on the Delta frequency as well would require consideration of all traffic-specific changes on the Delta frequency. Another aspect is to work on choosing the appropriate filtering.

\section{Acknowledgment}

This work was partially supported by the European Regional Development Fund in the Research Centre of Advanced Mechatronic Systems project, project number: CZ.02.1.01/0.0/0.0/16_019/0000867 within the Operational Programme Research, Development and Education.

\section{R E F E R E N C E S}

Asherson, P., Burton, C. L., Boomsma, D. I., Cormand, B., Dalsgaard, S., Franke, B., Gelernter, J. et al. (2018). Discovery of the First Genome-Wide Significant Risk Loci for Attention Deficit/Hyperactivity Disorder. Nature Genetics, 51(1), 63. doi: 10.1038/s41588-018-0269-7

Bartlett, M. S. (1950). Periodogram Analysis and Continuous Spectra. Biometrika, $37(1 / 2), 1-16$.

Bakhshi, A. D., Ahmed, A., Gulfarm, S. M., Khaqan, A. Yasin, S. Iqbal, S. Riaz, et al. (2013). Estimation of Baseline Wander Characteristics in ECG Signals Using Adaptive Transversal Filter and Lomb's Periodogram Analysis.Przeglad Elektrotechniczny, 89 (5), 107-110.

Bluschke, A., Roessner, V., \& Beste, C. (2016). Editorial Perspective: How to Optimise Frequency Band Neurofeedback for ADHD. Journal of Child Psychology and Psychiatry, 57(4), 457-461. doi: 10.1111/jcpp.12521 
Budzynski, T., H., Budzynski, H. K., Evans J. R., \& Abarbanel, A. (2009). Introduction to Quantitative EEG and Neurofeedback: Advanced Theory and Applications. 2nd Edition.

Clarke, A. R. , Barry, R. J., Karamacoska, D., \& Johnstone, S. J. (2019). The EEG Theta/Beta Ratio: A marker of Arousal or Cognitive Processing Capacity?. Applied Psychophysiology and Biofeedback, 44(2), 123-129. doi: 10.1007/s10484-018-09428-6

Hartmut, H., Strehl, U., Arns, M., Rothenberger, A., \& Ros, T. (2016). Neurofeedback in ADHD. Frontiers in Human Neuroscience, 6-21, 2016. doi: 10.3389/fnhum.2015.00602

Homan, R. W. Herman, J., \& Purdy, J. (1987). Cerebral Location of International 10-20 System Electrode Placement. Electroencephalography and Clinical Neurophysiology, 66(4), 376-382. doi: 10.1016/0013-4694(87)90206-9

Kawala-Janik, A., Zolubak, M., Bauer, W., Sobolewski, T., Nazimek, B., Sowa, M., \& Pelc, M. (2018). Implementation of Non-Integer Order Filtering for the Purpose of Disparities Detection in Beta Frequencies - A Pilot Study. 23rd International Conference on Methods and Models in Automation and Robotics (MMAR). doi: 10.1109/MMAR.2018.8486113

Kostick, K. (2017). ICD-10-CM Coding for Attention-Deficit/Hyperactivity Disorder (ADHD). Journal of AHIMA, 88(9), 56-59.

Kubacki, A., Sawicki, L., \& Owczarek, P. (2016). Detection of Facial Gestures Artefacts Created During an EEG Research Using Artificial Neural Networks. 21st International Conference on Methods and Models in Automation and Robotics. doi: 10.1109/MMAR.2016.7575236

Lazar, Z. I., Dijk, D.-J., \& Lazar, A. S. (2019). Infraslow Oscillations in Human Sleep Spindle Activity. Journal of Neuroscience Methods, 316, 22-34. doi: 10.1016/j.jneumeth.2018.12.002

Martinez-Murcia, F. J., Ortiz, A., Morales-Ortega, R., Lopez, P. J., Luque, J. L., Castillo-Barnes, D., Segovia, F. et al. (2019). Periodogram Connectivity of EEG Signals for the Detection of Dyslexia. Understanding the Brain Function and Emotions. LNCS, 11486, 350-359.

Mehran, Y. Z., Firoozabadi M., \& Rostami, R. (2015). Improvement of Neurofeedback Therapy for Improved Attention Through Facilitation of Brain Activity Using Local Sinusoidal Extremely Low Frequency Magnetic Field Exposure. Clinical EEG and Neuroscience, 46(2), 1-13. doi: 10.1177/1550059414524403

Moreno-Garcia, I., Delgado-Pardo, G., Camacho-Vara de Rey, C., MeneresSancho, S., \& Servera-Barcelo, M. (2015). Neurofeedback, Pharmacological Treatment and Behavioral Therapy in Hyperactivity: Multilevel Analysis of Treatment Effects on Electroencephalography.International Journal of Clinical and Health Psychology, 15(3), 217-225. doi: 10.1016/j.ijchp.2015.04.003

Moreno-Garcia, I., Meneres-Sancho, S., Camacho-Vara de Rey C., \& Servera, M. (2019). A Randomized Controlled Trial to Examine the Posttreatment Efficacy of Neurofeedback, Behavior Therapy, and Pharmacology on ADHD 
Measures. Journal of Attention Disorders, 23(4), 374-383. doi: 10.1177/108 7054717693371

Mostile, G., Giuliano, L., Monastero, R., Luca, A., Cicero, C. E., Donzuso, G., Dibilio, V. et al. (2019). Electrocortical networks in Parkinson's disease patients with Mild Cognitive Impairment. The PaCoS study. Parkinsonism E) Related Disorders, 64, 156-162. doi: 10.1016/j.parkreldis.2019.03.027

Mulkey, S. B., Kota, S., Govindan, R. B., Al-Shargabi, T., Swisher, C. B., Eze Jr., A., Hitchings, L. et al. (2019). The Effect of Labor and Delivery Mode on Electrocortical and Brainstem Autonomic Function During Neonatal Transition. Scientific Reports, 9(1). doi: 10.1038/s41598-019-47306-1

Rooney, I. M., \& Buck, J. R. (2019). Spatial Power Spectral Density Estimation Using a Welch Coprime Sensor Array Processor. The Journal of the Acoustical Society of America, 145(4), 2350. doi: 10.1121/1.5097572

Saad, J. F.,Kohn, M. R., Clarke, S., Lagopoulos, J., \& Hermens, D. F. (2018). Is the theta/beta EEG marker for ADHD inherently flawed? Journal of Attention Disorders, 22(9), 815-826. doi: 10.1177/1087054715578270

Schlerf, J. E., Galea, J. M., Spampinato, D. S., \& Celnik, P. A. (2015). Laterality Differences in Cerebellar-Motor Cortex Connectivity. Cerebral Cortex, 25(7), 1827-34.

Schmid, U., Schmid, K., \& Mall, V. (2018). FV 798. Neurofeedback Therapy for ADDH: Training with z-scored QEEG-Frequency Bands. Neuropediatrics, $49(\mathrm{~S} 02), \mathrm{S} 1-\mathrm{S} 69$.

Schonenberg, M., Wiedemann, E., Schneidt, A., Scheeff, J., Logemann, A., Keune, P. M., \& Hautzinger, M. (2017). Neurofeedback, Sham Neurofeedback, and Cognitive-Behavioural Group Therapy in Adults with Attention-Deficit Hyperactivity Disorder: a Triple-Blind, Randomised, Controlled Trial. The Lancet Psychiatry, 4(9), 673-684.

Sierra-Alonso, E. F., Antoni, J., \& Castellanos-Dominguez, G. (2019). Filtered Evelope Spectrum Using Short Periodograms for Bearing Fault Identification under Variable Speed. Advances in Mechanism and Machine Science, 73, 4157-66.

Skirrow, S., McLoughlin, G., Banaschewski, T., Brandeis, D., Kuntsi, J., \& and Asherson, P. (2015). Normalisation of Frontal Theta Activity Following Methylphenidate Treatment in Adult Attention-Deficit/Hyperactivity Disorder. European Neuropsychopharmacology, 25(1), 85-94.

Welch, P. (1967). The Use of Fast Fourier Transform for the Estimation of Power Spectra: a Method Based on Time Averaging over Short, Modified Periodograms. IEEE Transactions on Audio and Electroacoustics, 15(2), 70-73.

Wilhelm, I., Kurth, S., Ringli, M., Mouthon, A. L., Buchmann, A., Geiger, A., Jenni, O. G., \& Huber, R. (2014). Sleep Slow-Wave Activity Reveals Developmental Changes in Experience Dependent Plasticity. Journal of Neuroscience, 34(37), 12568-75. 
Initial Study on Implementation of the Low-Frequency Wave Markers...

World Health Organization (2015). International Classification of Diseases, Tenth Revision, Clinical Modification (ICD-10-CM). Retrieved from https://www. cdc.gov/nchs/icd/icd10cm.htm

Zhou, Y., Fang, K., Zhao, K., \& Ma, P. (2016). A Novel Credibility Quantification Method for Welch's Periodogram Analysis Result in Model Validation. In Proceedings of The 9th EUROSIM Congress on Modelling and Simulation - EUROSIM 2016, 142, 783-788. 